pulse scarcely to be counted. Says he knows he is dying, and asks to be taken home. Was removed by his parents, and taken a distance of about two miles in a cab. Did not complain of pain or fatigue on the journey ; conversed with his parents and brothers and sisters. Died at 10 P.M. ; intellect clear and tranquil to the last.

The patient was treated with iron, and later with iron and arsenic combined. He had a simple diet, and a small quantity of wine. His urine was examined almost daily; it averaged thirty-five ounces, was clear, acid, and free from albumen, and he passed from six to seven grammes of urea daily. The blood was examined several times; it always presented the characters described on admission.

Necropsy, at patient's home, forty-two hours after death.Body a good deal wasted, but not emaciated. Skin much paler than natural, but not so much so as during life ; it had a waxy appearance. Mucous membrane of mouth, nose, \&c., very anæmic. On opening body well-marked pamniculus adiposus was seen, the fat being of canary-yellow hue. Muscles of natural colour. Pericardium contained excess of clear straw-coloured fluid. Heart, not firmly contracted, larger than natural, apex formed by left rentricle. Both ventricles contained medium-sized clots of pale, reddishbrown colour, like meat jelly, and some fluid blood. Valves and orifices healthy; walls slightly thickened. Muscle of both ventricles of pale drab or fawn colour, with pale-yellow mottling (fatty degeneration). Lungs exceedingly pale; a little watery fluid could be squeezed out. Liver pale for the most part, but with patches of nutmeggy appearance. Spleen of natural size, firm and red. Kidneys : left very anæmic ; right venouslycongested; adrenals normal. Stomach thin, but not excessively so; mucous membrane congested and ecchymosed. Small intestine very thin, mucous membrane pale. Large intestine had well marked appendices epiploicæ; presented no changes except thimness. Pancreas natural. Mesenteric lands small. Retroperitoneal 1 rmphatic glands small and healthy-looking. Lymphatic glands of axilla natural. Thyroid gland rather large, but natural in appearance. Aorta of natural calibre, but very thin, pale, and inelastic: no changes in endarterium. Skull well shaped but thin. Brain exceedingly anæmic, otherwise normal. Periosteum removed from petrous bones: no discolouration or sign of disease. Orbits opened; contained abundant fat. Backs of eyes removed : hæemorrhage seen in retinæ. Pieces of clavicle and rib remored; marrow of a distinctly red colour.

Numeration of the blood-corpuscles in the fluid blood removed from the right ventricle was made some time after the necropsy by means of Dr. Gowers's hremacytometer. The number of coloured corpuseles was $1,940,000$ per cubic millimetre, or 38.9 per cent. of the natural number.

Remarks by Dr. MACKENZIE. - The case is a very characteristic example of idiopathic essential or progressive anæmia. The patient was, however, much below the age at which the disease is usually seen. No exciting cause could be discovered. No history of shock or fright was elicited. The diminution in the size of the coloured bloodcorpuscles (microcythæmia) was well-marked, but no nucleated corpuscles were detected. Hæmorrhagic extravasations into the retina are usually noticed in pernicions anæmia, and were well marked in this case. According to Litten, they have no diagnostic ralue in distinguishing this form of anxemia from others, for he has found retinal hromorrhages in anæemia from uterine cancer and hæmorrhage, menorrhagia, and hrematemesis. I have examined a very large number of eyes of persons suffering from cancer, chlorosis, and anzenia from other causes, without finding hæmorrhages; but, not having examined from the anæemia standpoint, I am not prepared to dispute Herr Litten's assertion. A point of much interest in the case was the presence of well-marked double optic neuritis. I am not aware of optic neuritis having been described, though I dare say it has been observed, by other observers in connexion with pernicious anæemia. Many (Centralblatt f. $d$. Med. Wissensch., 1875, s. 675 , in an account of a case, speaks of the papilla being deformed, and of the whole retina being cloudy; but it does not appear from his description of the ophthalmoscopic or microscopic appearances that there was neuritis. In my case the swelling of the disc was considerable, and the vessels were in places buried in exudation. The veins were very tortuous, as usual in neuritis, but of pale colour. with a broad light streak. The existence of optic neuritis caused hesitation in diagnosis in some who saw the case. Takenin conjunction with the pain in head, and discliarge from ear with deafness, it certainly suggested coarse cerebral disease. But the headache vas not so severe as is usual in intra. cranial tumour or abscess, and when first seen, although optic neuritis was present, there had been no purposeless vomiting. Moreover, the assumption of coarse intracranial disease did not explain the extreme anæmia. The occurrence of microcythremia assisted me to the diagnosis of progressive pernicious anæmia, and the subsequent progress of the case, the vomiting, febrile attacks without assignable cause, the recurring epistaxis, and bleeding of the gums confirmed me in my opinion. Arsenic, which has been so useful in Dr. Byrom Bramwell's hands, was administered in the form of Fowler's solution, without any amelioration of the symptoms. The post-mortem appearances were those usually observed. Dr. Wilks, than whom probably no one has had greater experience in this disease, says that usually no coagula are present in the heart. In this case clots were present in both ventricles, but quite peculiar in character. The enlargement of the heart (undoubted, though the organ could not be weighed) remains unexplained. The examination was made by candle-light. The marrow of the rib and clavicle was observed to be red. I have not yet examined it micro. scopically. The case will be published in detail when a histological examination of the retina and all the organs have been completed.

I am indebted to Mr. S. H. Fisher and Dr. Newton for many of the notes of the case, and for assistance in making the post-mortem examination.

\section{ROYAL INFIRMARY, EDINBURGH.} DEPRESSED FRACTURE OF FRONTAL BONE.

(Under the care of Professor SPENCE.)

Fon the notes of the following interesting case, we are indebted to Mr. T. F. Chavasse, M.B.

G. D-, aged eight, was admitted August 11th, 1877. Half an hour previously, while at play, he had fallen over the balustrade of a common staircase, and alighted upon his head on the pavement below.

On admission, a large irregular wound, with ragged edges, and the size of a five-shilling piece, was discovered half an inch above the right superciliary ridge. A portion of the frontal bone, nearly as large as the wound of the soft parts, was driven inwards upon the dura mater.

The child breathed quietly, and was unconscious, but could be roused. Chloroform having been administered Prof. Spence enlarged the wound of the skin somewhat, in an upward direction, carefully separated the periosteum from the depressed osseous portion, and then inserted an elevator between the dura mater and the bone, and raised those parts of the inner and outer tables of the skull which had been forced inwards, and took them away in three pieces. The pulsations of the brain became distinctly visible. The periosteum was folded over the dura mater, and the edges of the wound, as far as possible, were brought together with silver sutures.

Mr. Spence was of opinion that there was a fissure extend. ing towards the base of the skull, involving the orbital plate of the affected side.

The child remained unconscious all the night following the operation, but next morning he could be easily roused. The head was shaved, and ice continuously applied to it.

Aug. 13th. - There was considerable oedema of the upper eyelid of the right side, and the superficial and deep vessels of the eyeball were much injected.

A fortnight later a large abscess formed in the cornea of this eve, followed by sloughing of the cornea and also of a portion of the sclerotic. Eventually, however, a good stump of an eye remained.

The head injury progressed without a bad symptom. The portion of the dura mater that had been exposed gradually became covered with granulations, cicatrised, and contracted considerably. On Sept. 29 th the child was convalescent.

Presentation.-A very handsome inkstand, with the following inscription, was given to Mr. Moger, at Christ. mas, by the patients of the Highgate Dispensary, on his resignation of the duties of a surgeon to that institution :"Presented by the members of the Highgate Dispensary to R. G. Moger, Esq., F.R.C.S., in grateful recognition of his skilful and benevolent services to that institution during forty years. Dec. 1877." 